\title{
Traffic Jams and Shocks of Molecular Motors inside Cellular Protrusions
}

\author{
I. Pinkoviezky and N. S. Gov* \\ Department of Chemical Physics, The Weizmann Institute of Science, P.O. Box 26, Rehovot, Israel 76100
}

\begin{abstract}
Molecular motors are involved in key transport processes inside actin-based cellular protrusions. The motors carry cargo proteins to the protrusion tip which participate in regulating the actin polymerization, and play a key role in facilitating the growth and formation of such protrusions. It is observed that the motors accumulate at the tips of cellular protrusions, and in addition form aggregates that are found to drift towards the protrusion base at the rate of actin treadmilling. We present a one-dimensional driven lattice model, where motors become inactive after delivering their cargo at the tip, or by loosing their cargo to a cargo-less neighbor. The results suggest that the experimental observations may be explained by the formation of traffic jams that form at the tip. The model is solved using a novel application of mean-field and shock analysis. We find a new class of shocks that undergo intermittent collapses, and on average do not obey the Rankine-Hugoniot relation.
\end{abstract}

The traffic of molecular motors is an example of a nonequilibrium process [1. In order to describe the traffic of molecular motors the tools and theories of nonequilibrium statistical mechanics are useful [2]. In this letter we focus on the traffic of molecular motors inside actin-based cellular protrusions, such as filopodia and stereocilia. These protrusions are of a few microns in length and fractions of microns in diameter, and contain a polarized bundle of actin filaments 3 . The actin polymerizes at the protrusion tip, such that it provides the force for the protrusion initiation, and treadmills (retrograde flow) at a constant rate when the protrusion reaches a steady-state shape.

Unconventional myosins bind and move processively toward the protrusion tip on these filaments, as shown in Fig. 11a. Experiments analyzing myosin traffic revealed that motors accumulate over a finite length scale from the tip in protrusions of different lengths, for example see 4. A striking phenomenon is seen in a variety of experiments with different types of myosin motors [5] 7]: The traffic of motors exhibit wave-trains, or "pulses" of motor density, that move towards the base of the protrusion (opposite to the motors' motion). The velocity of these aggregates is found to be close to that of the actin retrograde flow, suggesting that these are inactive or jammed motors. The theoretical challenge for a successful model is to explain both the finite length of the accumulation of motors at the tips of the protrusions, and to provide a mechanism for the pulse-like counterpropagating aggregates of motors.

The simplest description of motors along a linear track is in terms of a Total Asymmetric Exclusion Process (TASEP) 2]. We can model a protrusion as a half closed tube, open at its base to the cell cytoplasm (Fig 1 a). Several works have dealt with this boundary condition (b.c.) together with attachment/detachment kinetics of the motors to the tracks [8-10]. These models find that at steady-state the tubes are practically all jammed, and

\footnotetext{
* Corresponding author: nir.gov@weizmann.ac.il
}

longer tubes have longer jammed (accumulation of motors) regions, in contrast with the observations for cellular protrusions. In [11] it was shown that a track coupled to an infinite reservoir produces an accumulation with a fixed length for different system sizes (see Fig.S1, [12]). However it is unlikely that the confined volume of a cellular protrusion can serve as an infinite reservoir.

Similarly, the properties of the observed pulse-like aggregates of motors do not fit the traffic jams arising in TASEP. For example, It seems from experiments that the aggregates originate only at the protrusion tips, are rather stable while propagating and have low density regions between them. This is not what happens in TASEP where jams appear at the high density (HD) phase [13, and jams appear everywhere. Sparse jams between free flow regions appear in models of vehicular traffic [14, but they result from the combination of synchronous update (a)

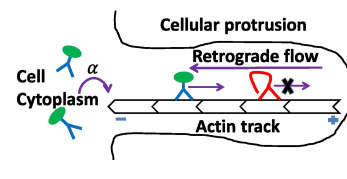

(b)

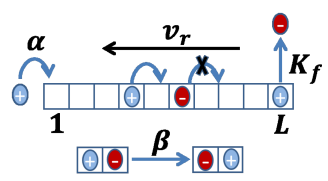

(c)

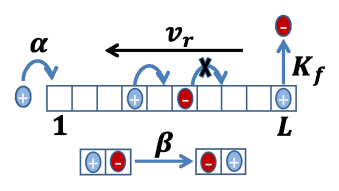

FIG. 1. (Color online) (a) An illustration of a cellular protrusion. Myosin motors enter with probability $\alpha d t$ from the cell's cytoplasm. They move on the actin track. Green ellipses are cargoes and without it the tail folds to inhibit the motor domain. (b) The model. Particles enter the first site with probability $\alpha d t$ an 'switch off' only at the last site; there is no exit from the left. The track itself moves backward with probability $v_{r} d t$. Particles can exchange their mobility state. (c) Particles interaction. Particles can exchange cargoes and change their mobility state. 
(a)

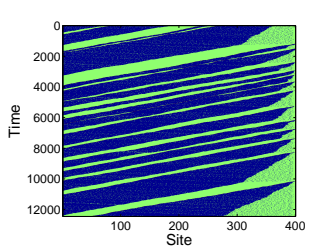

(b)

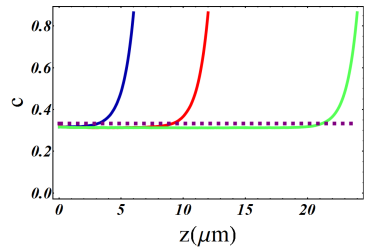

FIG. 2. (Color online) (a) Kymograph. Green (bright) points are active particles while dark blue is the empty space. (b) Density profiles for systems of lengths $z=6,12,24 \mu \mathrm{m}$. Data is for $\alpha=\beta=0.05, v_{r}=0.1$ and $K_{f}=0.6 / 300$. Dashed purple curve is the theoretical bulk density $c_{b}$ (Eq[2,S4 [12]).

and several particle velocities. There is no obvious reason why this should apply for molecular motors traffic.

To explain the observed phenomena we consider a generalization of TASEP as described in Fig. 1b. Each particle correspond to a molecular motor, and can be in one of two states: 'inactive' where it is immobile on the actin track, and 'active' where it can hop (move procssively along the actin filament). We note that internal degree of freedom was considered in the past [15, 18. Motivated by experiments and theoretical models, we propose the following properties for the dynamics of the activity state of the motors: It was found that several types of molecular motors become processive only when they are bound to a cargo molecule [19, 20]. Since in many cases the cargo is involved in regulating the actin polymerization at the protrusion tip [7, 21, 22], we assume that the motors can only detach from the cargo at the tip and become inactive. When inactive, motors may detach from the actin filament, or stay attached [19] and drift towards the protrusion base due to the retrograde flow. Furthermore, neighboring motors can "steal" the cargo from each other [20], and this introduces a conservation of the activity whereby an inactive motor can only become active at the expense of a motor jammed behind it (Fig 17). Such an interaction is known to produce a robust traffic-jam (condensate) 23. Note that there are other forms of interactions among motors that result in their inactivation 24, and therefore our model may be treated as a coarse-grained description of more complex set of underlying interactions.

The parameters in our model are: 1) $K_{f} d t$ is the probability for a particle to switch from active to an inactive state at the tip (at the last site). 2) $\alpha d t$ is the probability that a particle enters the system at the left boundary (from the cell cytoplasm). 3) $\beta d t$ is the probability that an inactive motor followed by an active motor switch their mobility state (fig. 1 1 ). 4) $v_{r}$ is the rate at which a new site (actin monomer) is added at the right end (tip) and simultaneously a site is removed at the left end. The actin retrograde velocity is therefore $v_{r}$, and we maintain a constant overall length of the protrusion which is assumed to be at steady-state. 5) We normalize the

(a)

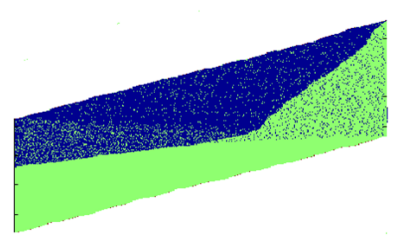

(b)

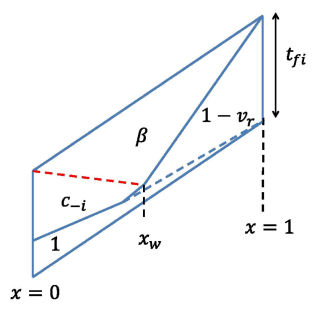

FIG. 3. (Color online) (a) An example of a parallelogram (or slice) for $\alpha=0.2, \beta=0.05, v_{r}=0.1$ and $\Lambda_{f}=0.2$. (b) A description of a slice with the different density regions and the slopes connecting them (see 12 for more examples). In both figures we see $x_{w}$ (Eq 1 where the densities $\left[c_{-}, \beta, 1-v_{r}\right]$ meet.

probabilities such that $d t$ is the hopping probability of an active particle.

The process defined above is the open b.c. version of a process with a single impurity particle on a ring [23]. In our process active motors enter from the left while impurity particles (inactive motors) enter from the right.

We show an example kymograph of the dynamics arising in our model in Fig. 2a. We find that near the tip there is a region of accumulation of motors, and large traffic jams are initiated there. Each traffic jam corresponds to an inactivation event of a motor at the tip, and these jams keep their size throughout the system as they move towards the left boundary. When a jam is formed, it transiently depletes the tip region, which gets refilled shortly afterwards. These properties of robust aggregates that form near the tip and deplete it, correspond qualitatively with the experimental observations of myosin-X [5] and myosin-XV [6] in filopodia .

We find in Fig. $2 \mathrm{~b}$ that the accumulation length of the motors near the tip is independent of the system size. The spatial extent of each site corresponds to a motor step (i.e. $30 \mathrm{~nm}$ ), therefore: $z=i \cdot 0.03 \mu m$ where $i$ is the site number. We stress that without the switching mechanism the track would be uniformly occupied with density $1-v_{r}$ except for a shock on the left end. The jams initiated by the inactive particles determine a finite length of the accumulation region and do not let it grow to the system size. Such localized accumulations of motors are observed near the tips of stereocilia of different lengths 4, and our model suggests that this arises from the turnover of motors through the formation of jams.

We now turn to analyze the model in detail, at steady state. The interesting behavior arises in the regime where the inactivation rate $K_{f}$ is small, and significant accumulation of motors occurs at the tip. It is convenient to use the so-called 'mesoscopic scaling' $K_{f}=\frac{\Lambda_{f}}{L}$ [16] and to use a spatial coordinate $x=\frac{i}{L}, 0 \leq x \leq 1$, where $L$ is the number of sites in the system.

We start by comparing the numerical simulations to a naive mean field approximation (MFA) 25], which fails, 


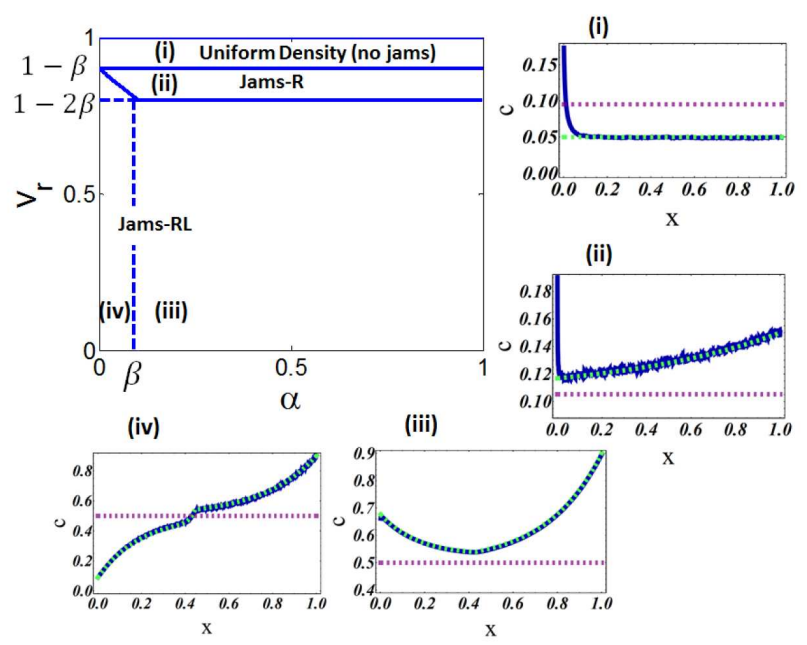

FIG. 4. (Color online) Phase diagram in $\left(\alpha, v_{r}\right)$ space and typical density profiles, using $\beta=0.1 . v_{r}>1-\beta$ : Uniform density phase (no jams), with density profile (i). $1-\beta>v_{r}>$ $1-\beta-c_{-}$: Jams-R phase where the behavior is dominated by the right boundary alone (density profile (ii)). $1-\beta-c_{-}>$ $v_{r}$ : Jams-LR phase where the behavior is influenced by both boundaries. The dashed vertical line separates this phase into two region: (iii) $\alpha>\beta$ and (iv) $\alpha<\beta$. Density profiles: Blue curves - simulation results; Green dashed curves - theoretical result of Eq2 Horizontal dashed purple curves - bulk density $c_{b}$.

as demonstrated in Fig. S2 [12]. The reason for this deviation lies in the fact that the system separates into regions of different mean densities and currents. We therefore proceed with a detailed calculation of the average concentration profiles of the motors in our model, which is based on using MFA within each distinct region. We divide the whole space-time evolution of the system into parallelogram slices as shown in Fig. 3a. The $i$ 'th parallelogram is defined by $t_{f i}$ - the time between two successive inactivation events at the tip.

We find four regions of different average concentrations (Fig 3 b, for more details see [12, Figs.S3,S4): (i) HD region near the tip, with density $1-v_{r}$, (ii) jammed regions of density 1 , (iii) free flow regions with density $\beta$, and (iv) the entrance region near the base with density $c_{-}=\min \left(\alpha, \frac{1-v_{r}}{2}\right)$. The lines separating these different regions have slopes that can be calculated according to the shock velocities [12, 26]. Using these slopes, we can find the meeting points between the different regions within the space-time slice. This allows us to calculate the time-duration that each spatial point spends in a region of certain density.

One such important triple meeting point is between the regions of densities $\left[1-v_{r}, \beta, c_{-}\right]$, which if it exists defines the meeting between the regions influenced by both boundaries (Fig. 3). If it does not exist as a real meeting point, it nevertheless defines the location of the matching between the left and right solutions. The location of this point is given by

$$
x_{w}=\frac{v_{r}\left(1-v_{r}-\beta-c_{-}\right)}{v_{r}\left(1-v_{r}-\beta-c_{-}\right)+\beta\left(1-c_{-}\right)}
$$

Summing the contributions of each region, we get that the average density profile is given by

$$
\begin{aligned}
x>x_{w}: & \frac{\beta}{c(x)}+\left(1-v_{r}-\frac{\beta}{\beta+v_{r}}\right) \exp \left(\frac{x-1}{\xi_{r}}\right) \\
x<x_{w}: & \\
c(x) & =\frac{\beta}{\beta+v_{r}}+\frac{v_{r}\left(c_{-}-\beta\right)}{\left(c_{-}+v_{r}\right)\left(\beta+v_{r}\right)} \exp \left(-\frac{x}{\xi_{l}}\right) \\
& +\left(1-c_{-}-\frac{c_{-}}{c_{-}+v_{r}}\right) \cdot \exp \left(\frac{\Lambda_{f}\left(1-v_{r}\right)\left(c_{-}-\beta\right)}{c_{-} \beta}\left(x_{w}-1\right)\right) \\
& \exp \left(\frac{\Lambda_{f}\left(1-v_{r}\right)\left(1-c_{-}\right)}{c_{-}}(x-1)\right)
\end{aligned}
$$

where

$$
\xi_{r}=\frac{\beta}{\Lambda_{f}(1-\beta)\left(1-v_{r}\right)}, \xi_{l}=\frac{v_{r}\left(1-v_{r}-\beta-c_{-}\right)}{\Lambda_{f}\left(1-c_{-}\right)(1-v r)(1-\beta)}
$$

$\xi_{l}, \xi_{r}$ are the 'healing' lengths of the left and right exponentials respectively as shown in Fig. 4(i)-(iii). The agreement between the simulations and the calculated density profile $(\mathrm{Eq}, 2)$ is very good, and improves for large systems $L \rightarrow \infty$. For comparison, we also denote the bulk density predicted from the periodic model [23] with retrograde flow: $c_{b}=\beta /\left(\beta+v_{r}\right)$ [12, as seen in Eq.2.

The jam size in the bulk is an exponential random variable with mean value

$$
\langle\Omega\rangle=L \frac{\beta}{\Lambda_{f}} \frac{1-v_{r}-\beta}{\left(1-v_{r}\right)(1-\beta)}
$$

We compare this result with simulation in Fig. S5 in [12] and we see that the two agree very well.

The phase diagram of the system, is shown in Fig. 4. We first note that for $\beta=1-v_{r}$, both the mean jam size (Eq 4 and the exponential accumulation at the tip (Eq2) vanishes. For $\beta>1-v_{r}$ we indeed find that the system does not exhibit any jams (except for the usual background of TASEP fluctuations), and the concentration is flat throughout the system at a value of $1-v_{r}$ (except for a shock at the left boundary, Fig 4 ). The system behave as TASEP with retrograde flow $v_{r}$ and zero current. This second-order transition can also be understood in terms of the velocities of the holes entering the system at the tip [12. Note that in the limit $v_{r}=0$, inactive particles will get stuck in the first site, preventing new particles from entering the system. This can be fixed by changing the b.c., so that the inactive particle is also removed at the left boundary.

Next, we note that $x_{w}$ becomes negative when: $1-$ $v_{r}-c_{-}<\beta$, which corresponds to a vanishing of the left exponential in $c(x)$, and therefore this phase is denoted as Jams-R (Fig 4 and profile (ii)). Finally for positive 
(a)

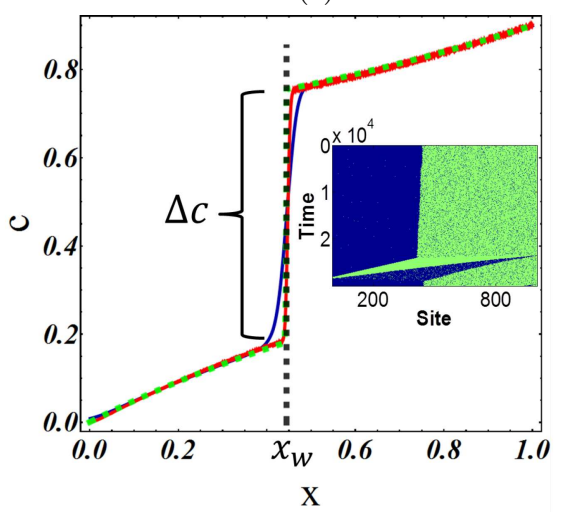

(b)

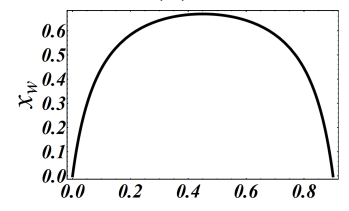

$(\mathrm{c})^{v_{r}}$

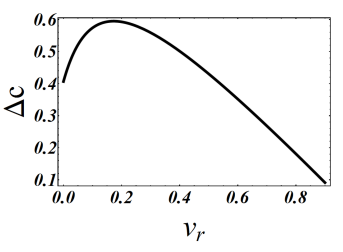

FIG. 5. (Color online) System exhibiting a shock: Density profile for $\alpha=1 / L, \beta=0.1, v_{r}=0.1, \Lambda_{f}=0.1$ with $L=10^{3}$ (blue curve) and $L=10^{4}$ (red curve). Green dashed line is the theoretical result of Eq. 2. Inset: Kymogrpah of the system. (b) The shock location $x_{w}$ from Eq1 and, (c) the density jump at the shock $(\Delta c$, Eq.S44).

$x_{w}$ (denoted as Jams-LR phase in Fig 4 ) we find that: for $\alpha>\beta$ jams grow as they approach the base (near the left end, Fig,4 iii), while for $\alpha<\beta$ they shrink (Fig, $4 \mathrm{v}$ ). This means that for $\alpha<\beta$ there is a step-like profile, with the step located at $x_{w}$. The steepness of the step is given by the exponential localized at $x_{w}$ in eq. (2). Taking the limit of $\alpha \rightarrow 0$ by using the scaling $\alpha \sim 1 / L$ and $L \rightarrow \infty$, results in the formation of a shock at $x_{w}$ (Fig. 5a). We find that the location of the shock in the system has a reentrant behavior as a function of $v_{r}$ (Fig. $5 \mathrm{p}$ ), while the amplitude of the density discontinuity ( $\Delta c$, Eq.S44) is also non-monotonic (Fig. 55).

Unlike previous shocks found in TASEP-like models 11, 16, 27, 28 the shock we find is only defined for the average concentration, while it maintains a dynamic nature: it undergoes intermittent collapses (kymograph in Fig. 5p. Furthermore, these shocks do not obey the usual Rankine-Hugoniot relation that follows from naive MFA: $c\left(x_{w}^{-}\right)=1-c\left(x_{w}^{+}\right)-v_{r}$. During the time that no motor is switched off at the tip, there is an accumulation of particles and a domain wall fulfilling the shock relation is established: $c\left(x_{w}^{-}\right)=0, c\left(x_{w}^{+}\right)=1-v_{r}$, so that: $\left.\Delta c\right|_{x_{w}}=1-v_{r}$. The probability that such a domain wall exists at any given time is given in Eq.S47, and is simply the fraction of time duration that no jam is initiated at the tip.

Discussion. Our model is able to reproduce two experimental observations of molecular motors in actinbased cellular protrusions, namely the finite accumulation length of the motors at the protrusions' tips, and the formation of backward-moving aggregates of motors from the tip to the base. In our model these phenomena are linked and both arise from the random process of trafficjam initiation at the tip, followed by the "relay-race"-like transport of the cargo between the motors. Note how-

ever, that this mechanism is maintaining individual motors near the tip, since only one motor is recycled back to the cytoplasm per traffic jam. Since there are multiple parallel actin tracks inside a real protrusion, we expect the turnover of motors to be more efficient than our onedimensional model suggests. The effects of such parallel tracks, as well as those of attachment/detachment kinetics will be explored in a future elaboration of this work.

Since the cargo carried by the motors is often involved in enhancing the actin polymerization at the tip [7, 21, 22, a full treatment of this system should include a feedback between the system size and motor/cargo distribution during the growth phase and the fluctuations around the steady-state length. While in the current work we considered a fixed geometry, we propose to investigate the feedback between motors and protrusion length in the future, similar to 2931 .

From the theory point of view we introduce here a model that has several unique features, compared to previous models of molecular motors on cytoskeletal tracks [29, 30, 32, 33]: (i) We find that the MFA fails, while it works well in separated domains of the system that are connected through shocks, (ii) we find that while there is a steady-state average density, the spatio-temporal behavior of the system is inherently dynamic exhibiting large fluctuations, (iii) a discontinuity (shock) can appear in the average steady-state density profile, but this shock is inherently dynamic and undergoes intermittent collapses.

Finally, we can make several qualitative predictions. Both the actin polymerization rate $\left(v_{r}\right)$ and the influx of motors $(\alpha)$ may be modified in experiments, and therefore the phase diagram of fig. 4 explored. We predict that increasing the retrograde flow will result in a decrease of the average jam size, as follows from eq. (4). The parameters $K_{f}, \beta$ are controlled by the cargo affinity to the motors: By modifying $\beta$ the system will change its phase according to Fig. 4a, and through $K_{f}$ the average size of the jams can be manipulated (eq. 4).

We gratefully acknowledge funding from the ISF (grant no. 580/12). This research is made possible in part by the historic generosity of the Harold Perlman Family. We thank Kirone Mallick and Tridib Sadhu for useful discussions.

[1] A. Kolomeisky and M. Fisher, Annu. Rev. Phys. Chem. 58, 675 (2007).

[2] T. Chou, K. Mallick, and R. Zia, Rep. Prog. Phys. 74, 116601 (2011).

[3] P. Mattila and P. Lappalainen, Nat. Rev. Mol. Cell Biol. 9, 446 (2008).

[4] M. E. Schneider, A. C. Dosé, F. T. Salles, W. Chang, F. L. Erickson, B. Burnside, and B. Kachar, J. Neurosci. 26, 10243 (2006).

[5] J. Berg and R. Cheney, Nat. Cell Biol. 4, 246 (2002).

[6] I. Belyantseva, E. Boger, S. Naz, G. Frolenkov, J. Sellers, 
Z. Ahmed, A. Griffith, and T. Friedman, Nat. Cell Biol. 7, 148 (2005).

[7] F. Salles, R. Merritt, U. Manor, G. Dougherty, A. Sousa, J. Moore, C. Yengo, A. Dosé, and B. Kachar, Nat. Cell Biol. 11, 443 (2009).

[8] M. J. Müller, S. Klumpp, and R. Lipowsky, J. Phys. Cond. Mat. 17, S3839 (2005).

[9] M. Naoz, U. Manor, H. Sakaguchi, B. Kachar, and N. S. Gov, Biophys. J. 95, 5706 (2008).

[10] P. Zhuravlev, Y. Lan, M. Minakova, and G. Papoian, Proc. Natl. Acad. Sci 109, 10849 (2012).

[11] A. Parmeggiani, T. Franosch, and E. Frey, Phys. Rev. Lett. 90, 086601 (2003).

[12] See online supplemental information for more details on the calculations.

[13] G. Schutz, in Phase Transitions and Critical Phenomena, Vol. 19, edited by C. Domb and J. L. Lebowitz (New York: Academic, New York, 2000).

[14] M. Schreckenberg, A. Schadschneider, K. Nagel, and N. Ito, Phys. Rev. E 51, 2939 (1995).

[15] K. Nishinari, Y. Okada, A. Schadschneider, and D. Chowdhury, Phys. Rev. Lett. 95, 118101 (2005).

[16] T. Reichenbach, T. Franosch, and E. Frey, Phys. Rev. Lett. 97, 50603 (2006).

[17] S. Klumpp, Y. Chai, and R. Lipowsky, Phys. Rev. E 78, 041909 (2008).

[18] I. Pinkoviezky and N. S. Gov, New J. Phys. 15, 025009 (2013).

[19] N. Umeki, H. Jung, T. Sakai, O. Sato, R. Ikebe, and
M. Ikebe, Nat. struct. Mol. Biol. 18, 783 (2011).

[20] U. Manor, M. Grati, C. M. Yengo, B. Kachar, and N. S. Gov, BioArchitecture 2, 171 (2012).

[21] H. Tokuo and M. Ikebe, Biochem. Biophys. Res. Comm. 319, 214 (2004).

[22] U. Manor, A. Disanza, M. Grati, L. Andrade, H. Lin, P. P. Di Fiore, G. Scita, and B. Kachar, Cur. Biol. 21, 167 (2011).

[23] K. Mallick, J. Phys. A: Math. Gen. 29, 5375 (1996).

[24] O. Quintero, J. Moore, W. Unrath, U. , F. Salles, M. Grati, B. Kachar, and C. Yengo, J. Biol. Chem. 285, 35770 (2010).

[25] B. Derrida, E. Domany, and D. Mukamel, J. Stat. Phys. 69, 667 (1992).

[26] A. B. Kolomeisky, G. M. Schütz, E. B. Kolomeisky, and J. P. Straley, J. Phys. A: Math. Gen. 31, 6911 (1998).

[27] H. Hinsch and E. Frey, Phys. Rev. Lett. 97, 95701 (2006).

[28] C. Arita, J. Bouttier, P. Krapivsky, and K. Mallick, arXiv preprint arXiv:1307.4367 (2013).

[29] A. Melbinger, L. Reese, and E. Frey, Phys. Rev. Lett. 108, 258104 (2012).

[30] K. Sugden, M. Evans, W. Poon, and N. Read, Phys. Rev. E 75, 031909 (2007).

[31] K. Sugden and M. Evans, J. Stat. Mech. 2007, P11013 (2007).

[32] R. Lipowsky, S. Klumpp, and T. Nieuwenhuizen, Phys. Rev. Lett. 87, 108101 (2001).

[33] D. Johann, C. Erlenkämper, and K. Kruse, Phys. Rev. Lett. 108, 258103 (2012). 